\title{
Oceanic crust accumulation at the CMB and its interactions with a primordial reservoir
}

\author{
Timothy D. Jones ${ }^{1}$, NATE Sime ${ }^{1}$, Peter VAN KeKeN ${ }^{1}$
}

${ }^{1}$ Department of Terrestrial Magnetism, Carnegie Institution for Science, Washington DC, USA.

tjones@carnegiescience.edu

Former oceanic crust and primordial material are both identified as source components of ocean island basalts (OIBs). Their coexistence in the lavas of individual volcanic events suggests that a strong degree of mixing occurs prior to eruption. Here we aim to quantify this mixing in geodyanmical simulations to constrain how much of the compositional diversity recorded in OIBs is due to inherent source heterogeneity.

We present new high resolution models with dynamically forming plates under a yield stress rheology. Our study explores the conditions under which oceanic crust is sequestered to the core-mantle-boundary (CMB), and how this accumulating reservoir interacts with one that is primordial. We statistically capture the compositional heterogeneity within mantle reservoirs that feed plumes and constitutes the geochemical source of OIBs. 